\title{
Living with an inferior sinus venosus defect
}

\author{
Varda Singhal, Shane F. Tsai, Jonathan Cramer, Yiannis S. Chatzizisis \\ Department of Cardiovascular Medicine, University of Nebraska Medical Center, \\ Omaha, NE, United States
}

\begin{abstract}
A 70-year-old female was admitted to hospital with worsening exertional dyspnea. Her symptoms initially started 7-8 years ago. Previous transthoracic echocardiography evaluations demonstrated right heart dilation, mild pulmonary hypertension and no evidence of an intracardiac shunt. During admission, cardiac catheterization demonstrated elevated right heart cardiac output, mild pulmonary hypertension and normal pulmonary vascular resistance. There was a significant increment in oxygen saturation within the right atrium $(68 \%$ at inferior vena cava, $85 \%$ at right atrium) with a Qp/Qs of 2.65. Cardiac magnetic resonance imaging (Fig. 1A) showed an absence of the secundum atrial septum originating at the mouth of the inferior vena cava (inferior vena cava/atrial straddle) consistent with an inferior sinus venosus defect. Follow-up transesophageal echocardiography (Fig. 1B) confirmed a $1.6 \mathrm{~cm}$ inferior sinus venosus defect. Transesophageal echocardiography bubble study
\end{abstract}

with Valsalva was negative with arm injection, but positive with injection through a lower extremity. Retrospective review of non-contrast computed tomography scan (years prior), done to evaluate lung parenchyma in the setting of unexplained pulmonary hypertension, did show the complex defect (Fig. 1C). The patient underwent uncomplicated surgical patch closure of the defect and reported improvement of symptoms at 1 month.

Sinus venosus defects account for $5-10 \%$ of atrial septal defects with inferior defects being very rare. The complex nature of the interatrial septum coupled with poor subcostal transthoracic echocardiography windows and a low suspicion for congenital defects in older adults presents a significant diagnostic challenge and often leads to delays in diagnosis. A careful assessment of the entire interatrial septum using advanced imaging techniques is always warranted for accurate diagnosis and treatment of unexplained right side dilation.

Conflict of interest: None declared

Address for correspondence: Yiannis S. Chatzizisis, MD, PhD, Department of Cardiovascular Medicine, University of Nebraska Medical Center, 982265 Nebraska Medical Center, Omaha, NE, 68198 USA, tel: (402) 559-5156, fax: (402) 559-8355, e-mail: ychatzizisis@icloud.com 


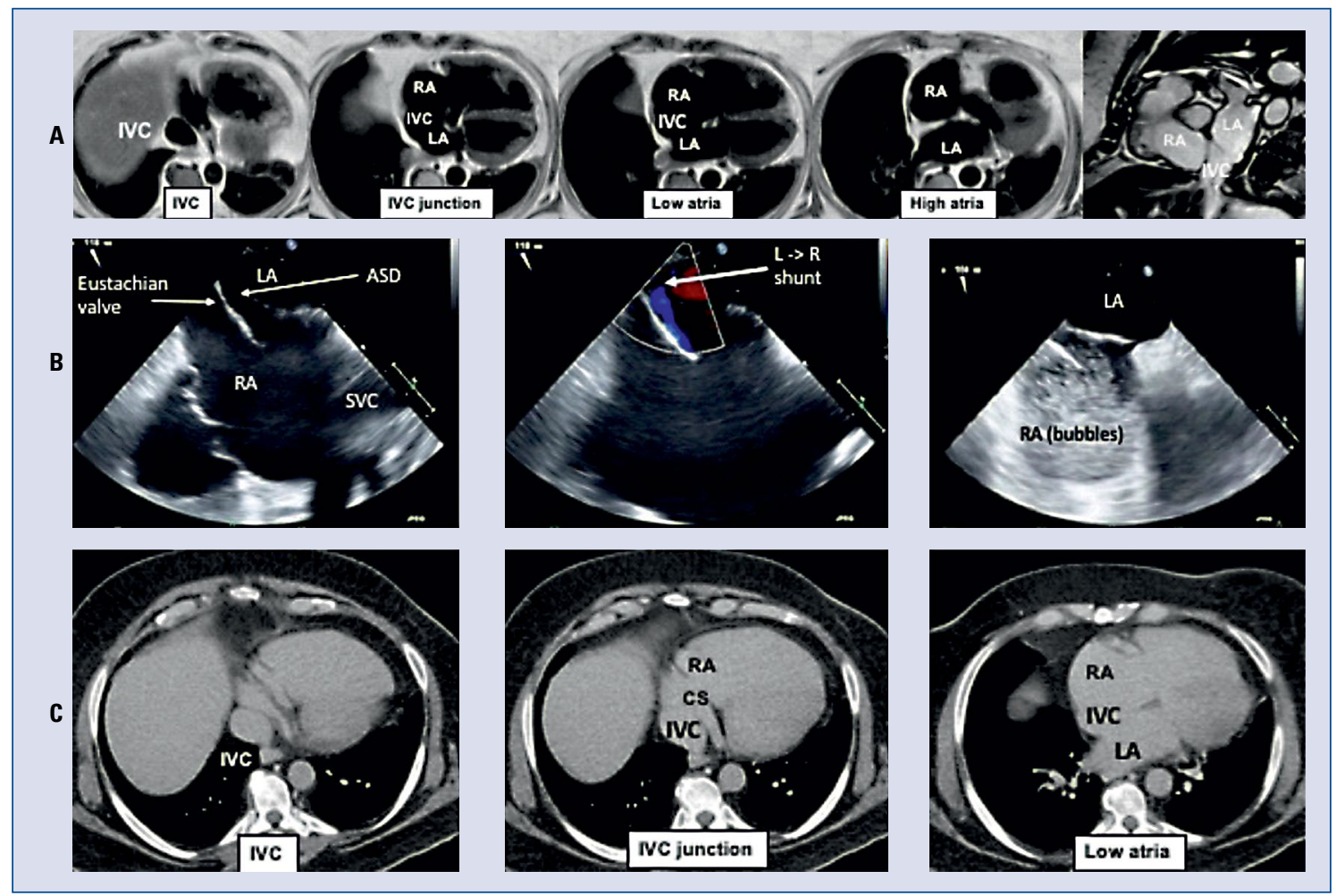

Figure 1. Inferior sinus venosus by cardiac magnetic resonance imaging (A), transesophageal echocardiography (B) and computed tomography (C); ASD — atrial septal defect; CS — coronary sinus; IVC — inferior vena cava; LA — left atrium; RA — right atrium; SVC — superior vena cava. 